3

\title{
Fast microplate assay for simultaneous determination of thiols and dissolved sulfides in wastewater
}

Bruno Coulomb $^{1 *}$, Fabien Robert-Peillard ${ }^{1}$, Edwin Palacio ${ }^{2}$, Robert Di Rocco ${ }^{1}$, Jean-Luc Boudenne $^{1}$

${ }^{1}$ Aix Marseille Univ, CNRS, LCE UMR7376, 13331 Marseille, France.

${ }^{2}$ Laboratory of Environmental Analytical Chemistry. University of the Balearic Islands, E07122 Palma de Mallorca, Illes Balears, Spain.

* corresponding author: Tel: +33 413551028 - Fax: +33 413551060

Email address: bruno.coulomb@univ-amu.fr

\section{Abstract}

The present paper reports the development and the application of a microplate assay for the fast and individual determination of thiols and sulfides. The method is based on the reaction of ethyl propiolate with thiols and sulfides, yielding thioacrylates absorbing in the UV range. Thioacrylate generated by derivation of sulfide with EP presents a bathochromic shift of maximal absorbance wavelength of $16 \mathrm{~nm}$ compared to thioacrylates formed with thiols. EP concentration, reaction time and derivatization $\mathrm{pH}$ were carefully optimized in order to separately quantify thiols and sulfides using a spectral deconvolution procedure in 15 minutes. The detection limits were 0.6 and $2.8 \mu \mathrm{M}\left(63.7\right.$ and $\left.92.4 \mu \mathrm{g} . \mathrm{L}^{-1}\right)$ for mercaptopropionic acid (used as reference thiol compound for calibration) and dissolved hydrogen sulfide respectively. Non-thiol sulfur compounds (methionine, sulfates and sulfites) or other nucleophile compound did not display any interference in the determination of MPA or sulfides. Interference from metals was suppressed with application of a reduction step with TCEP and DTPA. Finally, the method has been successfully applied to the analysis of raw and treated wastewater. 
1 Keywords: thiols; ethyl propiolate; hydrogen sulfide; high-throughput analysis; UV spectral deconvolution.

\section{Introduction}

Malodorous emissions and odor control in collection and treatment of wastewater remain a major concern for operators of wastewater treatment plants (WWTP). Organic and inorganic compounds, directly or indirectly responsible for odors, are emitted by sewage or result from the anaerobic degradation of organic matter containing sulfur or nitrogen. Among these compounds, the two main inorganic odorous compounds are hydrogen sulfide and ammonia [1], but we can also mention thiols, indoles and other inorganic or organic nitrogen and sulfur compounds. Complex mixtures of sulfur compounds at low concentrations (range from $\mu \mathrm{g} . \mathrm{m}^{-3}$ to $\mathrm{mg} \cdot \mathrm{m}^{-3}$ ) form the majority of these malodorous emissions associated with sewage treatment works. Hydrogen sulfide $\left(\mathrm{H}_{2} \mathrm{~S}\right)$, carbon disulfide $\left(\mathrm{CS}_{2}\right)$, methyl mercaptan or methanethiol $\left(\mathrm{CH}_{3} \mathrm{SH}\right)$, dimethyl sulfide $\left[\left(\mathrm{CH}_{3}\right)_{2} \mathrm{~S}\right]$ and dimethyl disulfide $\left[\left(\mathrm{CH}_{3}\right)_{2} \mathrm{~S}_{2}\right]$ were the most frequently identified compounds in influent and primary effluent of a WWTP [2,3].

Thiol compounds are often quantified by separation methods like gas chromatography coupled to flame photometric detector [4] or mass spectrometer [3], or like high performance liquid chromatography (HPLC) with pre-column derivatization coupled to fluorescence [5-8], UV detection [9] or mass spectrometry [10]. Several studies have also focused on the global quantification of thiol levels mainly in biological systems by absorbance [11,12], fluorescence measurement $[13,14]$ or enzymatic acitvity [15]. Many easy-to-use microplate kits, usually based on the use of the Ellman's method or fluorescent maleimide derivatives and calibrated using standard solutions of glutathione and/or cysteine, are commercialized for the measurement of free or total thiols in biological samples. Traditional Ellman's reagent can also be used for inorganic sulfides determination and numerous other analytical techniques reported 
1 for hydrogen sulfide determination in natural waters or sewage may be mentioned including

2 spectrophotometry [16,17], fluorimetry [18], HPLC coupled to UV-Vis detector [19],

3 inductively coupled plasma-atomic emission spectroscopy [20] or flow systems [21,22].

4 Many analytical systems exist for hydrogen sulfide analysis in the gas phase. However, it is 5 also important to monitor dissolved sulfides and thiols so as to predict $\mathrm{H}_{2} \mathrm{~S}$ emissions at 6 facilities such as sewage treatment plants or to control the quantities of chemical inhibitors to 7 add to a wastewater stream. Some authors have developed methodologies based on multiwavelengths exploitation of UV-Vis spectra for the determination of sulfides in natural waters [23] or dissolved organic sulfide (DOS) in industrial wastewater [24], but these techniques require $\mathrm{pH}$ buffering of samples and suffer from relatively high detection limits.

Recently, Owen (2008) has described the reaction of thiol compounds with esters of propiolic acid (like ethyl propiolate, EP) under mild conditions to form stable and strongly UV absorbing thioacrylates [25]. Tzanavaris et al. (2010) have adapted this derivatization method as a sequential injection analysis system for the determination of thiols in pharmaceutical samples [26]. Even though these two studies demonstrated good selectivity of this derivation method, our preliminary tests showed that sulfides could also react with ethyl propiolate to form a stable derivative with a red shift of the maximum absorption wavelength of about twenty nanometers as compared to thiols derivatives (Fig. S1).

In the present work, we decided to optimize the derivatization properties of ethyl propiolate (EP) to develop a new and simple spectrophotometric high-throughput assay in a 96-well plate for simultaneous determination of thiols and sulfides in wastewater. The assay was validated by comparison with Ellman's and OrthoPhtalAldehyde-AminoEthanol (OPA-AE) methods on real wastewater samples.

\section{Experimental}




\subsection{Reagents and solutions}

All chemicals used in this study were of analytical reagent grade. Ethyl propiolate, sodium sulfide nonahydrate, mercaptopropionic acid, mercaptoacetic acid, dimethyldisulfide, ethanethiol, tris(2-carboxyethyl)phosphine hydrochloride (TCEP) and diethylene triamine pentaacetic acid (DTPA) were purchased from Sigma-Aldrich (Saint-Quentin Fallavier, France), cysteine, glutathion, 2-mercaptoethanol, o-phthaldialdehyde and 5,5'-dithiobis(2nitrobenzoic acid) (DTNB) were from Alfa-Aesar (Schiltigheim, France). EP and other thiol compounds stock standard solutions and working solutions were prepared in ultrapure water (Millipore, resistivity $>18 \mathrm{M} \Omega \mathrm{cm}$ ).

\subsection{Instrumentation}

Microplate absorbance and fluorescence measurements were carried out on a microplate reader (Infinite M200, Tecan France SAS, Lyon, France) controlled by i-control ${ }^{\mathrm{TM}}$ software (Tecan) in clear polystyrene 96 flat-bottom well microplates for absorbance or black polystyrene $96 \mathrm{~V}$ bottom wells shape microplates for fluorescence (Fisher Scientific, Illkirch, France).

\subsection{Analysis of thiols and dissolved sulfides}

A protocol for simultaneous determination of thiols (R-SH) and sulfides (HS ${ }^{-}$) has been developed at the microplate level. In addition, a reduction step by TCEP has enabled the determination of total thiols (R-SH + R-S-S-R'+R-S-Me-S-R', with Me a metal and R and R' a functional group) by measuring variation of signal after and before reduction. To compare the performance of this analytical method, thiols determination was also performed with two other methods traditionally used for this purpose and optimized in microplate format: OPA-AE derivatization method and Ellman's method. The various protocols are described below. 
2.3.1. High throughput assay for reduced and total thiols, and dissolved sulfides determination by EP derivatization

The reduction of total thiols was carried out by adding $10 \mu 1$ of TCEP $20 \mathrm{mM}$ in a buffer solution (0.2 M borate $\mathrm{pH} 9.5$ and DTPA $2 \mathrm{mM}$ ) to $100 \mu \mathrm{L}$ of sample or standard. The plate was shaken and after $8 \mathrm{~min} 50 \mu \mathrm{L}$ of EP $50 \mathrm{mM}$ in $0.2 \mathrm{M}$ borate buffer $\mathrm{pH} 9.5$ was added and absorbance spectrum was measured between 230 and $350 \mathrm{~nm}$ after $2 \mathrm{~min}$ for total thiols determination and 15 min for dissolved sulfides determination.

The same protocol can be used for reduced thiols determination by directly dispensing $100 \mu \mathrm{L}$ of sample into the wells of the microplate, without the reduction step by TCEP. Both protocols are summarized in Scheme 1.Quantification of total or reduced thiols and dissolved sulfides was carried out by spectral deconvolution using ACD/Spectrus Processor software (ACD/Labs 2012, Totonto, Canada) and the SIMPLISMA (SIMPLe-to-use Interactive Self-modeling Mixture Analysis) tool, based on a two-stepped regression procedure using the normalized spectral intensities [27].

\subsubsection{Reduced and total thiols determination by OPA-AE method}

In this paper, the OPA-AE method was adapted and optimized for microplate analysis [28] and analytical protocol was as follows: $15 \mu \mathrm{L}$ of sample or standard solutions was dispensed into the wells of the microplate, where $145 \mu \mathrm{L}$ of a reagent solution (AE $26 \mathrm{mM}$ and DTPA $20 \mathrm{mM}$ in $0.05 \mathrm{M}$ borate buffer $\mathrm{pH} 9.9$ ) and $130 \mu \mathrm{L}$ of OPA $50 \mu \mathrm{M}$ in ethanol were then added. Reduced thiols determination was carried out by fluorescence intensity measurement after shaking for 7 min, with wavelengths set at $330 \mathrm{~nm}$ and $455 \mathrm{~nm}$ for excitation and emission respectively. The analytical protocol was also adapted to total thiols measurement: during the first step, 15 $\mu 1$ of sample was first mixed with $15 \mu 1$ of TCEP $20 \mathrm{mM}$. After a shaking time of 6 min, the analytical protocol was continued as described in the previous paragraph. 
Inorganic sulfides do not interfere in thiol compounds measurement with this OPA-AE method (Fig. S2).

\subsubsection{Reduced thiols determination by Ellman's reagent}

The determination of reduced thiols was also performed using Ellman's method adapted to a microplate measurement. Briefly, $100 \mu \mathrm{l}$ of sample or standard solution was mixed with 100 $\mu \mathrm{L}$ of a DTNB reagent $(1 \mathrm{mg} / \mathrm{ml}$ in $200 \mathrm{mM}$ phosphate buffer $\mathrm{pH} 8)$. The absorbance was measured at $412 \mathrm{~nm}$ after incubation for 15 minutes.

\section{Results and discussion}

\subsection{Thiols and sulfides derivatization}

As displayed in Supplementary data (Fig. S1), the maximum absorption wavelength of the products either formed between EP and a thiol compound such as mercaptopropionic acid (MPA) or with sulfide ions is found at $288 \mathrm{~nm}$ and $304 \mathrm{~nm}$, respectively. These wavelengths have been selected for the course of this study. We deliberately chose MPA as the reference thiol compound herein because this compound has an important environmental significance [29] and possesses one of the higher pKa of thiol compounds [25], therefore requiring higher $\mathrm{pH}$ value than other thiols to reach optimal reaction yield (reaction scheme between MPA and EP is presented in Fig. S3).

\subsection{1. $\mathrm{pH}$ optimization}

Thiols derivatization with EP is usually carried out at basic $\mathrm{pH}$ to increase the reaction rate [30]. In the same time, it is well known that EP can react with other nucleophiles such as amines to yield UV absorbing adducts [25,31]. Although these interfering compounds usually react slowly with EP, they can potentially cause serious interferences in the case of environmental 
samples with a wide range of concentrations for these compounds. Careful choice of the $\mathrm{pH}$ value during derivation step can limit these interferences.

Fig. 1 displays the evolution of absorbance of EP derivatives as a function of $\mathrm{pH}$ value, formed with i) MPA for reference thiol compound, ii) hydrogen sulfide and iii) glycine as reference amine compound.

The absorbance of MPA derivative was stable at $\mathrm{pH}$ above 10, which is consistent with the acidity of the thiol group for MPA $(\mathrm{pKa}=10.3)$ that allows reaction of MPA with EP through its thiolate group. Regarding sulfides, the optimum $\mathrm{pH}$ for the reaction was between 9 and 9.5, which suggests that EP reacts with the HS ${ }^{-}$form of sulfides. Finally, as expected, glycine shows higher response at $\mathrm{pH}$ around 10.5.

In this study, a $\mathrm{pH}$ value of 9.5 was thus selected as the best compromise for maximizing the response of sulfides and thiols and minimizing interference of amines.

\subsubsection{Effect of EP concentration and reaction time}

EP concentration has obviously a significant influence on the reaction yield. The effect of EP concentration was studied in the 1-75 mM range for MPA and hydrogen sulfide at $\mathrm{pH} 9.5$. The experimental results are shown in Fig. 2 and Fig. 3.

EP concentration greater than $10 \mathrm{mM}$ had no significant impact on the derivatization yield of MPA (Fig. 2), as demonstrated in previous studies concerning other thiols of biological or environmental interest $[25,26,32]$. On the other hand, it can be seen that absorbance of the EP derivative with $\mathrm{HS}^{-}$is highly dependent on the concentration of EP. Hydrogen sulfide needs a large excess of EP to reach significant yields, probably due to the fact that hydrogen sulfide has a lower nucleophicity than thiols. A concentration of $50 \mathrm{mM}$ of EP was thus the best compromise for the derivatization of MPA and sulfides and was selected for further 
experiments. Glycine response was also tested at this concentration of EP and was still very low (absorbance $<0.05)$.

Time-dependence of derivatization has been assessed by acquiring absorbance versus time during the reaction between EP and MPA or hydrogen sulfide. Experiments were carried out with a $50 \mathrm{mM}$ EP solution (Fig. 3). Optimal reaction times were 2 minutes for the derivatization of MPA (thereby limiting the cross interference of sulfides) and 15 minutes for the derivatization of sulfides and were therefore selected for the following experiments.

\subsubsection{Oxidized thiols}

It is well known that thiol group can easily complex a wide range of metals in environmental samples. Trace metals such as $\mathrm{Cu}^{2+}, \mathrm{Cd}^{2+}, \mathrm{Hg}^{2+}, \mathrm{Pb}^{2+}$ and $\mathrm{Zn}^{2+}$ have particularly high affinity for SH groups, and we noticed that some complexes between thiols and metals almost completely quenched subsequent reaction with $\mathrm{EP}\left(\mathrm{Cu}^{2+}\right.$ and $\mathrm{Fe}^{3+}$ especially). Moreover, thiols can also be oxidized and form disulfide bond, which cannot be detected by direct reaction with EP. To quantify total thiols content of a sample, the analytical protocol should therefore include a reducing step to unbind metals from thiols or cleave disulfide bonds. Dithiothreitol (DTT) is one of the most used agent for reducing thiols. Its use is however limited because DTT is not stable due to air oxidation and must be kept cool and under an inert atmosphere. In recent years, TCEP was used as thiols reducing agent [32,33] and offered many advantages such as an improved stability in aqueous solution and a reactivity in a wider $\mathrm{pH}$ range than DTT.

Preliminary studies on quantification of MPA when bound with $\mathrm{Cu}^{2+}$ ions showed that TCEP alone was insufficient to correctly quantify the concentration of MPA due to $45 \%$ yield of bond cleavage (Fig. S4). Quantification could only be achieved after a reduction step by TCEP in the presence of DTPA as complexing agent with a release of $97 \%$ of free MPA (Fig. S4). In this sense, some experiments were carried out in order to optimize amount concentration of TCEP 
during reduction step while a concentration of 2 mM DTPA was kept constant. The reducing efficiency of TCEP was evaluated by spiking a $50 \mu \mathrm{M}$ MPA solution with $160 \mu \mathrm{M} \mathrm{Cu} \mathrm{Cu}^{2+}$. Increasing concentrations of TCEP were added to the spiked MPA solution in the presence of 2 mM DTPA and left to react 6 minutes (Fig. 4.A). Experimental results showed that more than 97\% of MPA was released within 6 minutes with a TCEP amount concentration between 20 and $40 \mathrm{mM}$. Reaction yield of reduction by TCEP was estimated by acquiring absorbance of EP-MPA derivative as a function of time when reduction carried out with $20 \mathrm{mM}$ TCEP and 2 mM DTPA (Fig. 4.B). A reaction time of 8 minutes was sufficient to quantify total MPA content. Some experiments were also conducted to optimize the concentration of DTPA. The results showed that a concentration of 2 mM DTPA was sufficient to effectively bind $\mathrm{Cu}^{2+}$ and a higher DTPA concentration resulted in an increase of blank signal. A final concentration of $20 \mathrm{mM}$ TCEP and $2 \mathrm{mM}$ DTPA with a reaction time of 8 minutes were selected for final analytical protocol and further experiments.

\subsection{Analytical parameters}

The analytical parameters were defined using the optimal analytical protocols. The calibration curve obtained for MPA determination was Absorbance $=(0.006 \pm 0.0001) \mathrm{C}+(0.0267 \pm 0.003)$, where Absorbance was measured at $288 \mathrm{~nm}$ and and $\mathrm{C}$ was the MPA concentration in $\mu \mathrm{M}$ $\left(\mathrm{R}^{2}=0.9986 ; \mathrm{n}=7\right.$ triplicates). Calibration curve was linear on the range $0-500 \mu \mathrm{M}$. The calibration curve obtained for sulfides determination was Absorbance $=(0.0105 \pm 0.0004) \mathrm{C}+$ (0.0105 \pm 0.005$)$, where Absorbance was measured at $304 \mathrm{~nm}$ and $\mathrm{C}$ was the sulfide concentration in $\mu \mathrm{M}\left(\mathrm{R}^{2}=0.9962 ; \mathrm{n}=7\right.$ triplicates $)$. Calibration curve was linear on the range 0 $250 \mu \mathrm{M}$. The detection limits $(3 \sigma, \mathrm{n}=10)$ were 0.6 and $2.8 \mu \mathrm{M}\left(63.7\right.$ and $\left.92.4 \mu \mathrm{g} . \mathrm{L}^{-1}\right)$ for MPA and sulfide respectively and the quantification limits $(10 \sigma, \mathrm{n}=10)$ were $2.1 \mu \mathrm{M}\left(222.9 \mu \mathrm{g} . \mathrm{L}^{-1}\right)$ for MPA and $7.3 \mu \mathrm{M}\left(240.9 \mu \mathrm{g} . \mathrm{L}^{-1}\right)$ for sulfide. The relative standard deviations (RSD) were 
calculated from 10 successive standard determinations $(100 \mu \mathrm{M})$ and reproducibility of $1.8 \%$ and $1.9 \%$ was obtained for MPA and sulfide respectively.

In Table 1, main analytical features of our analytical protocols and previously developed methods are compared. The present work shows that the developed method has a detection limit among the lowest with a 2 minutes reaction time compared to 5 to 60 minutes for other methods. NAM method [13] has a similar detection limit $(1 \mu \mathrm{M})$ but requires a 60 minutes reaction time and the response of various thiols is not uniform. The method also suffers from interference of sulfites [34]. PAPAIN method [15] has the best detection limit and sensitivity but requires degassing of solutions beforehand for 1 hour. The 4-DPS method displays improved reactivity and sensitivity compared to the Ellman's method with a limited reaction time [12]. However, it does not differentiate thiols and sulfides. The OPA-AE method adapted from the study of Mopper and Delmas [8] has better analytical features but has the major drawback, as the NAM method, to present a very variable reactivity for the different thiols (Fig. 5). Fig. 5 depicts the relative reactivity of various thiols based on MPA as a reference. The EP method displays good homogeneity in the response of the various thiols, like Ellman's method, while the OPA-AE method displays a rather poor response for thiols such as GSH or MAA. The homogeneity of the signals for various thiols enables general application of our methodology to quantify the overall thiols.

For sulfides, the methods based on redox reactions are fast but suffers from interferences from other reducing compounds such as nitrites [17]. Three methods compared in Table 1 present fairly similar analytical performance. The advantage of the EP method developed here is to separately quantify thiols and sulfides with the same derivatizing reagent in 15 minutes.

\subsection{Interferences}


1 The potential interference of other compounds on the determination of MPA and sulfide by the

2 proposed methods was studied. Series of solutions containing $100 \mu \mathrm{M}$ of MPA or $100 \mu \mathrm{M}$ of

3 sulfide were prepared with addition of potential interfering compounds selected for their

4 presence in environmental samples. It has been assumed that an element did not interfere when

5 the variation of resulting concentration of MPA or sulfide was lower than $\pm 10 \%$ (Table 2).

6 We first evaluated the cross interferences between MPA and sulfides. Thanks to the spectral 7 deconvolution (see 2.3.1), each compound can be determined selectively in the presence of a 8 50-fold excess of the other analyte. We also evaluated the potential interferences resulting from non-thiol sulfur compounds (reduced or oxidized) including methionine (in the range 0.5-3 $\mathrm{mM})$, sulfates $(20 \mathrm{mM})$ and sulfites $(5 \mathrm{mM})$. Results indicate that these non-thiol compounds do not lead to a significant change in the response of MPA or sulfides when they are present in realistic ratios compared to targeted analytes, even for amino acids such as methionine or glycine that could potentially lead to serious interference if experimental conditions were not optimized. Other significant potential nucleophiles such as halides, thiocyanate or acetate (50 $\mathrm{mM}$ each) were also evaluated and displayed no interferences in the determination of MPA or sulfides. Finally, interference from metals such as $\mathrm{Cu}^{2+}(160 \mu \mathrm{M}), \mathrm{Fe}^{3+}(180 \mu \mathrm{M})$ and $\mathrm{Zn}^{2+}(160$ $\mu \mathrm{M})$ was less than $6 \%$ with application of the reduction step with TCEP and DTPA.

\subsection{Validation}

Considering the possible matrix interference in real samples, determination of thiols and sulfides in water samples was first validated by spiking real samples with different amounts of MPA (for reduced thiols), cystine (for total thiols) and sodium sulfide (for dissolved sulfides). This standard addition method has already been used in some studies about the determination of thiol compounds [36,37]. The proposed method was applied to the determination of reduced and total thiols and sulfides in raw and outlet wastewater spiked with $25 \mu \mathrm{M}$ of MPA, cystine 
and sodium sulfide. The recovery study of the targeted analyte was performed in triplicates. Results were compared to recoveries obtained with Ellman's method. Results indicated a good recovery of targeted analytes and an absence of matrix effect on the tested samples. The recoveries of reduced thiols were $96.4 \pm 2.1 \%$ in raw wastewater and $95.1 \pm 1.3 \%$ in outlet with developed method. Ellman's method gave recoveries of $96.1 \pm 1.9 \%$ and $98.2 \pm 2.6 \%$ for reduced thiols on the same samples. The recoveries of total thiols were $98.3 \pm 1.6 \%$ and $96.6 \pm 0.9 \%$ in raw wastewater and outlet respectively. The determination of sulfides showed also good recoveries too with $98.5 \pm 1.7 \%$ and $102.3 \pm 0.8 \%$ in raw wastewater and outlet respectively.

4. Conclusion

The development of a microplate assay for the fast and individual determination of thiols and sulfides has been successfully optimized in this study. Separated quantification of thiols and sulfides has been achieved by exploitation of the absorbance shift of the derivatives of the targeted analytes with ethyl propiolate. Total thiols can also be measured with an innovative combination of a reducing agent (TCEP) and a complexing agent (DTPA). To the best of our knowledge, this is the first method that can simultaneously quantify free thiols, total thiols (bound to metals or in the oxidized form) and sulfides in aqueous samples, with a very acceptable reaction time and low limits of detection. The method was successfully validated by application to raw and treated wastewater and proved its applicability to real environmental samples.

\section{Acknowledgement}

This research was supported by the French Research Agency (ANR), programme “EMERGENCE” (ANR-10-EMMA-038-01). 


\section{References}

[1] R.R. Dague, Fundamentals of odor control, J. Water Pollut. Control Fed. 44 (1972) 583595.

[2] Y. Hwang, T. Matsuo, K. Hanaki, N. Suzuki, Identification and quantification of sulfur and nitrogen containing odorous compounds in wastewater, Wat. Res. 29 (1995) 711-718.

[3] H. Van Langenhove, K. Roelstraete, N. Schamp, J. Houtmeyers, GC-MS identification of odorous volatiles in wastewater, Wat. Res. 19 (1985), 597-603.

[4] P.R. Bérubé, P.D. Parkinson, E.R. Hall, Measurement of reduced sulphur compounds contained in aqueous matrices by direct injection into a gas chromatograph with a flame photometric detector, J. Chromatogr. A. 830 (1999), 485-489.

[5] T. Toyo'oka, S. Uchiyama, Y. Saito, K. Imai, Simultaneous determination of thiols and disulfides by high-performance liquid chromatography with fluorescence detection, Anal. Chim. Acta, 205 (1988) 29-41.

[6] R.A.Winters, J. Zukowski, N. Ercal, R.H. Matthews, D.R. Spitz, Analysis of glutathione, glutathione disulfide, cysteine, homocysteine, and other biological thiols by high-performance liquid chromatography following derivatization by n-(1-pyrenyl) maleimide, Anal. Biochem. 227 (1995), 14-21.

[7] K. Mopper, D. Delmas, Trace determination of biological thiols by liquid chromatography and precolumn fluorometric labeling with o-phthalaldehyde, Anal. Chem. 56 (1984) 2557 $2560,1984$.

[8] C. Parmentier, P. Leroy, M. Wellman, A.J. Nicolas, Determination of cellular thiols and glutathione-related enzyme activities: versatility of high performance liquid chromatographyspectrofluorimetric detection, Chromatogr. B. 719 (1998) 37-46. 
1 [9] K. Kusmierek, G. Chwatko, R. Glowacki, P. Kubalczyk, E. Bald, Ultraviolet derivatization

of low-molecular-mass thiols for high performance liquid chromatography and capillary electrophoresis analysis, J. Chromatogr. B. 879 (2011), 1290-1307.

[10] B. Seiwert, U. Karst, Simultaneous LC/MS/MS determination of thiols and disulfides in urine samples based on differential labeling with Ferrocene-based Maleimides, Anal. Chem., 79 (2007) 7131-7138.

[11] G.L. Ellman, Tissue sulfhydryl groups, Arch. Biochem. Biophys. 82 (1959), 70-77.

[12] I.O. Egwin, H.J. Gruber, Spectrophotometric measurement of mercaptans with 4,4'dithiopyridine, Anal. Biochem. 288 (2001) 188-194.

[13] E. Hatakeyama, N. Matsumoto, T. Ochi, T. Suzuki, H. Ohrui, H. Meguro, Fluorometric determination of thiol and disulfide groups in protein using $\mathrm{N}$-(9-acridinyl)maleimide, Anal. Sci. 5 (1989) 657-661.

[14] Y. Yang, X. Guan, Rapid and thiol-specific high-throughput assay for simultaneous relative quantification of total thiols, protein thiols, and nonprotein thiols in cells, Anal. Chem. 87 (2015) 649-655.

[15] R. Singh, W.A. Blättler, A.R. Collinson, An amplified assay for thiols based on reactivation of papain, Anal. Biochem. 213 (1993) 49-56.

[16] A. Svenson, A rapid and sensitive spectrophotometric method for determination of hydrogen sulfide with 2,2'-dipyridyl disulfide, Anal. Biochem. 107 (1980) 51-55.

[17] B. Shyla, G. Nagendrappa, New spectrophotometric methods for the determinations of hydrogen sulfide present in the samples of lake water, industrial effluents, tender coconut, sugarcane juice and egg, Spectrochim. Acta Part A. 96 (2012) 776-783.

[18] Y. Yang, C. Yin, F. Huo, J. Chao, Y. Zhang, Highly selective relay recognition of hydrogen sulfide and $\mathrm{Hg}(\mathrm{II})$ by a commercially available fluorescent chemosensor and its application in bioimaging, Sens. Actuators, B. 204 (2014) 402-406. 
1 [19] D. Tang, P.H. Santschi, Sensitive determination of dissolved sulfide in estuarine water by

2 solid-phase extraction and high-performance liquid chromatography of methylene blue, J.

3 Chromatogr. A. 883 (2000) 305-309.

4 [20] M. Colon, J.L. Todoli, M. Hidalgo, M. Iglesias, Development of novel and sensitive

5 methods for the determination of sulfide in aqueous samples by hydrogen sulfide generation-

6 inductively coupled plasma-atomic emission spectroscopy, Anal. Chim. Acta 609 (2008) 160-

$7 \quad 168$

8 [21] A. Safavi, M. Ali Karimi, Development of novel and sensitive methods for the determination of sulfide in aqueous samples by hydrogen sulfide generation-inductively coupled plasma-atomic emission spectroscopy, Talanta 57 (2002) 491-500.

[22] M.A. Spaziani, J.L. Davis, M. Tinani, M.K. Carroll, On-line determination of sulfide by the 'MethyleneBlueMethod' with diode-laser-based fluorescence detection, Analyst 122 (1997) $1555-1557$

[23] E.A. Guenther, K.S. Johnson, K.H. Coale, Direct ultraviolet spectrophotometric determination of total sulfide and iodide in natural waters, Anal. Chem. 73 (2001) 3481-3487. [24] B. Roig, E. Chalmin, E. Touraud, O. Thomas, Spectroscopic study of dissolved organic sulfur (DOS): a case study of mercaptans, Talanta 56 (2002) 585-590.

[25] T.C. Owen, Thiol detection, derivatization and tagging at micromole to nanomole levels using propiolates Bioorg. Chem. 36 (2008) 156-160.

[26] P.D. Tzanavaras, A. Tsiomlektsis, C.K. Zacharis, Derivatization of thiols under flow conditions using two commercially available propiolate esters, J. Pharm. Biomed. Anal. 53 (2010) 790-794.

[27] W. Windig, J. Guilment, Interactive self-modeling mixture analysis, Anal. Chem., 63 (1991) 1425-1432. 
1 [28] E. Palacio-Barco, Développement d'une méthode alternative pour la caractérisation

2 fonctionnelle par spectrofluorescence des composés organiques dissous dans les écosystèmes, PhD thesis, Aix-Marseille University, 2010.

[29] P. Salgado, T. Visnevschi-Necrasova, R.P. Kiene, I. Azevedo, A.C.S. Rocha, C.M.R. Almeida, C. Magalhaes, Determination of 3-Mercaptopropionic acid by HPLC: a sensitive method for environmental applications, J. Chromatogr. B 992 (2015) 103-108.

[30] P.D. Halphen, T. C. Owen, Carboxyalthioacrylates, J. Org. Chem. 38 (1973), 3507-3510. [31] W. Bottomley, The reaction of amines with methyl propiolate, Tetrahedron Lett., 21 (1967) 1997-1999.

[32] C.K. Zacharis, P.D. Tzanavaras, A. Zotou, Ethyl propiolate as a post-column derivatization reagent for thiols: Development of a green liquid chromatographic method for the determination of glutathione in vegetables, Anal. Chim. Acta 690 (2011) 122-128.

[33] J.C. Han, G.Y. Han, A procedure for quantitative determination of tris(2carboxyethyl)phosphine, an odorless reducing agent more stable and effective than dithiothreitol, Anal. Biochem. 220 (1994) 5-10.

[34] E. Taylan, H. Resmi, The analytical performance of a microplate method for total sulfhydryl measurement in biological samples, Turk J. Biochem. 35 (3), 275-278, 2010.

[35] K. Akasaka, T. Suzuki, H Onrui, H. Meguro, Fluorometric determination of sulfite with N-(9-Acridinyl)-maleimide for High Performance Liquid Chromatography, Agric. Biol. Chem. 50 (1986), 1139-1144.

[36] Z. Wang, D.M. Han, W.P. Jia, Q.Z. Zhou, W.P. Deng, Reaction-based fluorescent probe for selective discrimination of thiophenols over aliphatic thiols and its application in water samples, Anal. Chem. 84 (2012) 4915-4920.

[37] J.V. Ros-Luis, B. Garcia, D. Jimenez, R. Martinez-Manez, F. Sancenon, J. Soto, F Gonzalvo, M.C.J Valldecabres, Squaraines as fluoro-chromogenic probes for thiol-containing 
1 compounds and their application to the detection of biorelevant thiols, Am. Chem. Soc. 126

2 (2004) 4064-4065.

3

4

5

6

7 


\section{$1 \quad$ Figures and Tables}

2

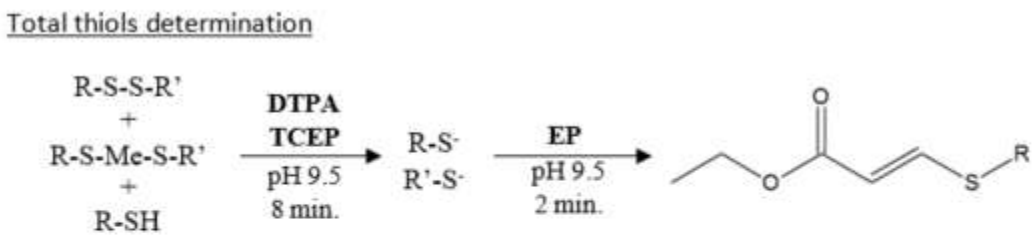

Reduced thiols and dissolved sulfides determination

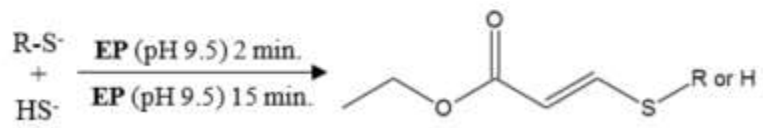

3

4 Scheme 1 Schematic depiction of the protocol for determination of total thiols, reduced thiols 5 and disolved sulfides.

6

7

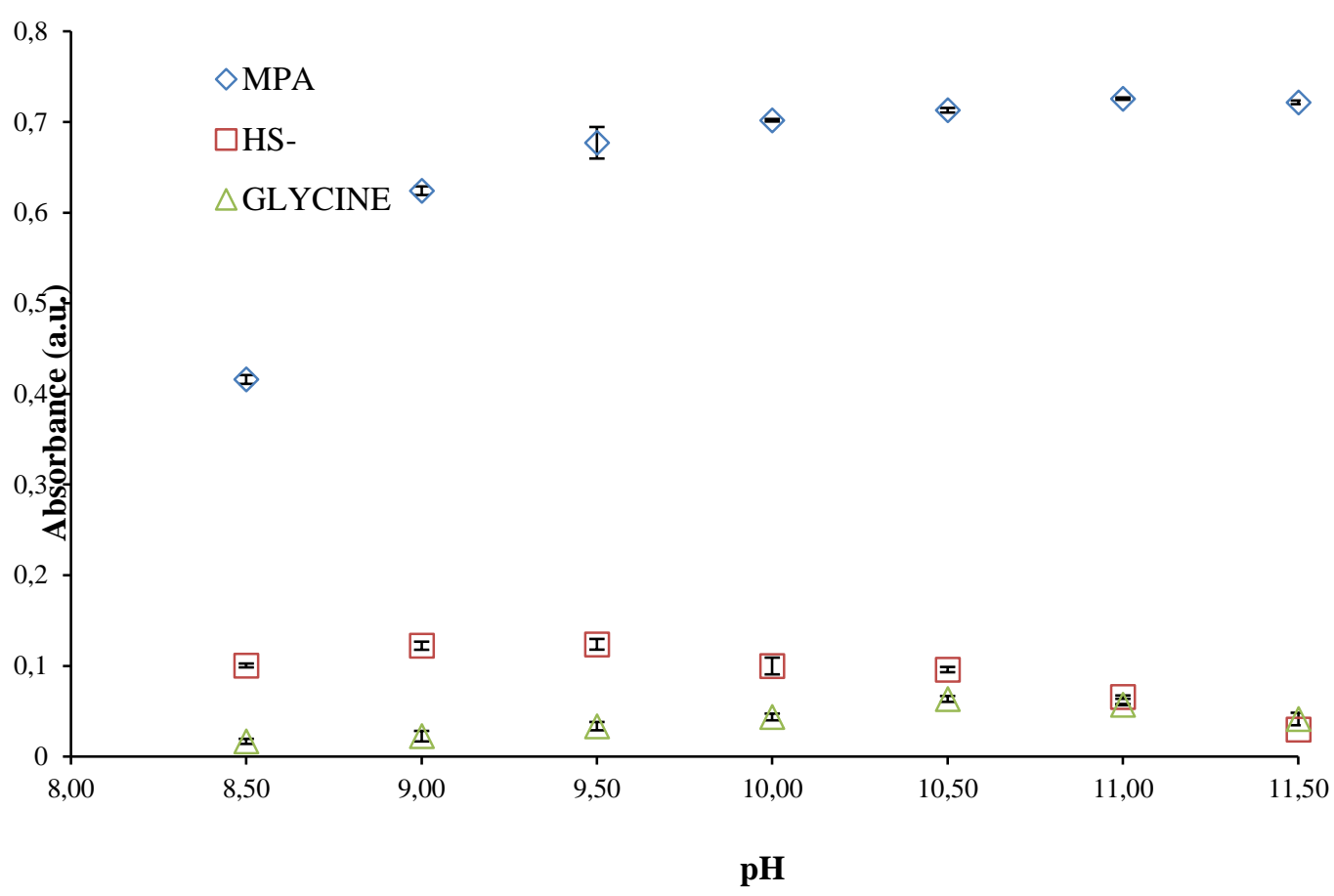

8

9 Fig. 1 Evolution of absorbance (at $288 \mathrm{~nm}$ for MPA (diamonds) and glycine (triangles); at 304 $\mathrm{nm}$ for hydrogen sulfide (squares)) as a function of $\mathrm{pH}$ value during derivatization step. MPA, 
$1 \mathrm{HS}^{-}$and glycine were at $100 \mu \mathrm{M}$ each. Derivatization was carried out in duplicate with EP at 5

$2 \mathrm{mM}$ during 5 minutes, $\mathrm{pH}$ was buffered by borate or phosphate.

3

4

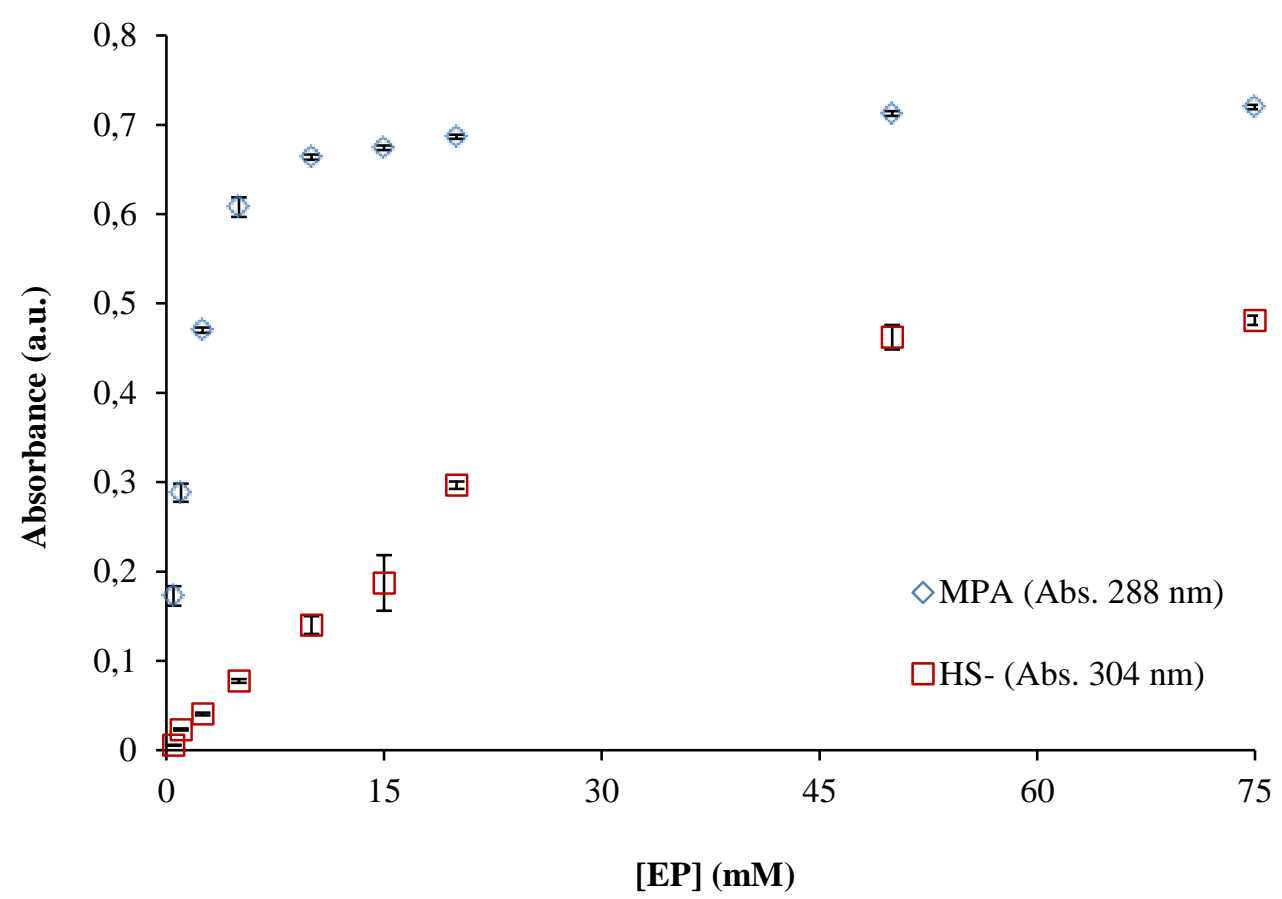

5

6 Fig. 2 Evolution of the absorbance (at $288 \mathrm{~nm}$ for MPA (diamonds); at $304 \mathrm{~nm}$ for $\mathrm{HS}^{-}$

7 (squares)) as a function of EP concentration. MPA and $\mathrm{HS}^{-}$were at $100 \mu \mathrm{M}$ each. Derivatization

8 was carried out in duplicate during 5 minutes, $\mathrm{pH}$ was buffered at 9.5 by borate.

9 


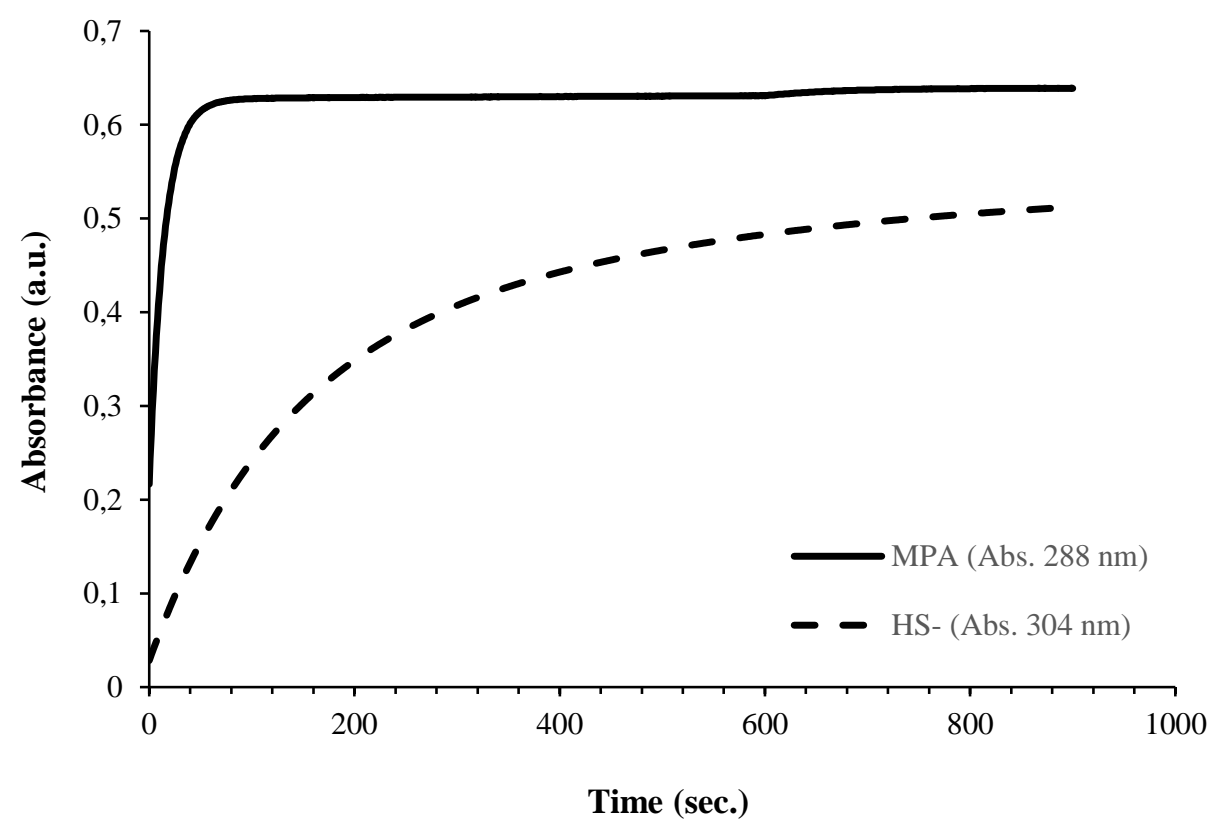

1

2 Fig. 3 Evolution of absorbance (at $288 \mathrm{~nm}$ for MPA (solid line); at $304 \mathrm{~nm}$ for hydrogen sulfide

3 (dash line)) as a function of time. EP was at $50 \mathrm{mM}$. MPA and hydrogen sulfide were at 100

$4 \quad \mu \mathrm{M}$ each. $\mathrm{pH}$ was buffered at 9.5 by borate.

5

6

7

8

9

10

11

12

13

14

15 


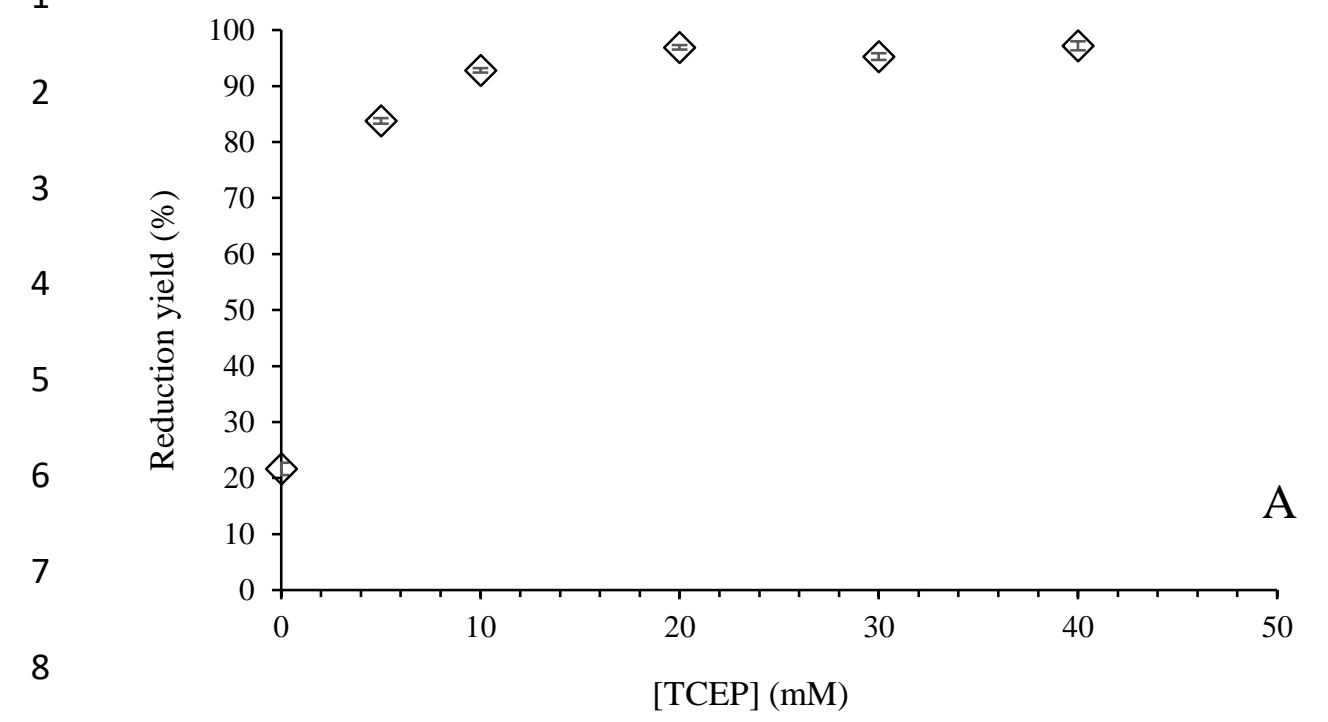

9

10

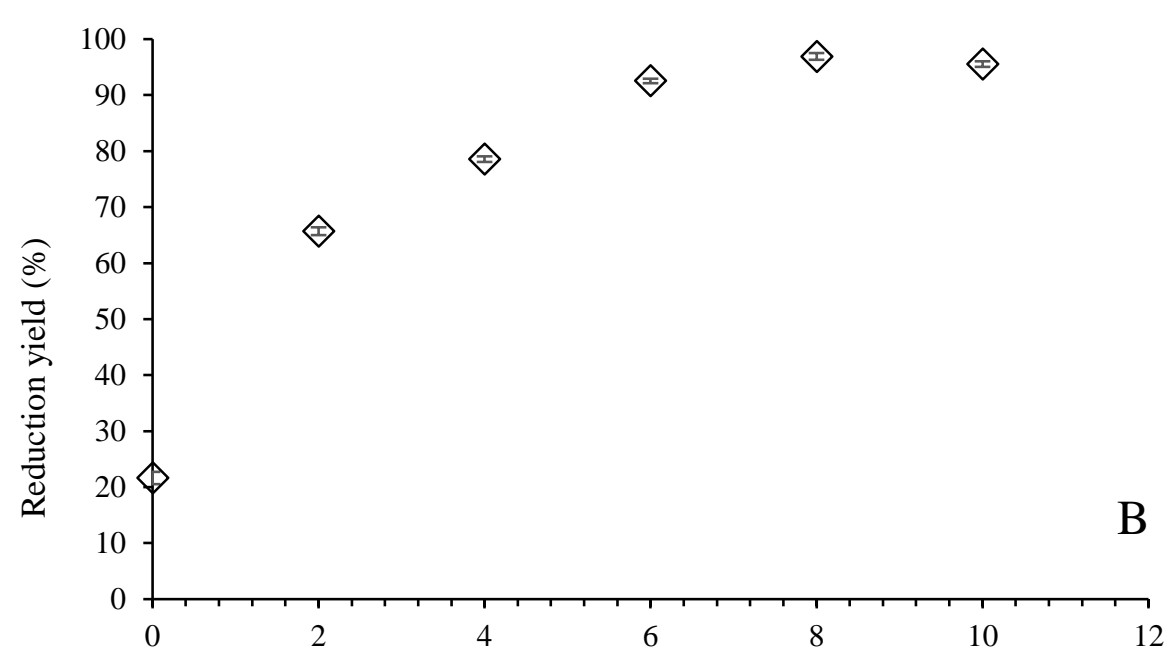

17

18

Time (min)

Fig. 4 Influence of various concentrations of TCEP with 6 min reduction time on reduction yield (Fig 4A); Influence of reduction time with $20 \mathrm{mM}$ TCEP on reduction yield (Fig 4B). Derivatization of $50 \mu \mathrm{M}$ MPA in the presence of $160 \mu \mathrm{M} \mathrm{Cu}^{2+}$ was carried out in duplicate with $50 \mathrm{mM}$ EP during 2 minutes, $\mathrm{pH}$ was buffered at 9.5 by borate. 


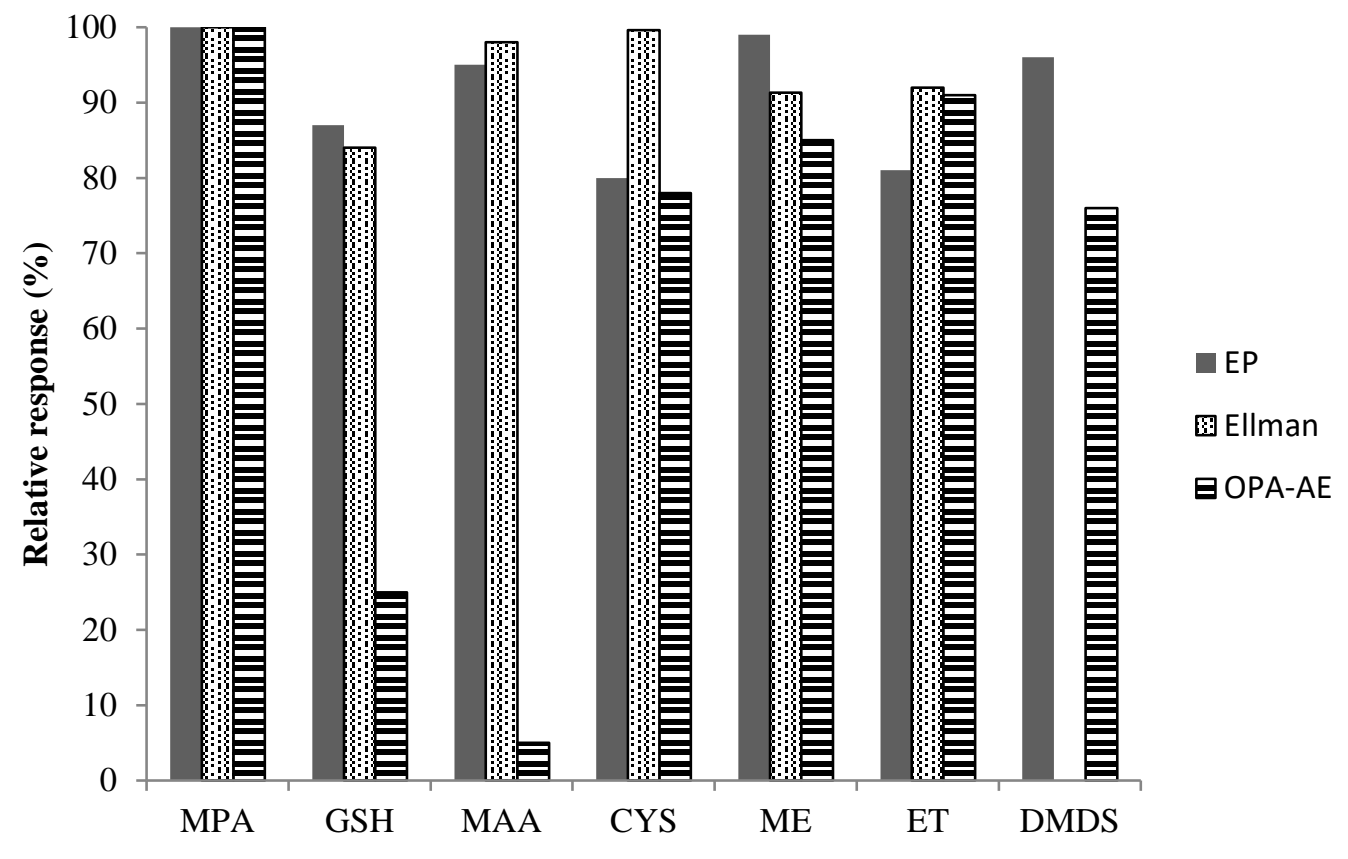

2 Fig. 5 Relative response of some thiol compounds compared to MPA (reference base 100) after

3 derivatization by EP following proposed method (grey plain), by Ellman's reagent (grey 25\%)

4 or by OPA-AE method (horizontal lines). The concentration of tested thiols was $100 \mu \mathrm{M}$ each.

5 GSH: glutathione - OPA: Ortho-phtalaldehyde - AE: Aminoethanol - MAA: mercaptoacetic

6 acid-CYS: cysteine - ME: mercaptoethanol-ET: ethanethiol - DMDS: dimethyldisulfide 
Table 1 Main analytical features of some studies on free thiols and sulfides determination.

NAM: N-(9-acridinyl)maleimide - 4-DPS : 4,4'-dithiodipyridine - PAPAIN: cysteine protease - 2-PDS: 2,2' dipyridil disulfide

\begin{tabular}{|c|c|c|c|c|c|c|c|c|c|}
\hline Analyte & & & & iiols & & & & Sulfides & \\
\hline $\begin{array}{l}\text { Method } \\
\text { (detection) }\end{array}$ & $\begin{array}{l}\text { 4-DPS } \\
\text { (UV) }\end{array}$ & $\begin{array}{l}\text { NAM } \\
\text { (fluo) }\end{array}$ & $\begin{array}{l}\text { PAPAIN } \\
\text { (Vis) }\end{array}$ & $\begin{array}{l}\text { DTNB } \\
\text { (Vis) }\end{array}$ & $\begin{array}{l}\text { OPA-AE } \\
\text { (fluo) }\end{array}$ & $\begin{array}{l}\text { EP } \\
\text { (UV) }\end{array}$ & $\begin{array}{l}\text { 2-PDS } \\
\text { (UV) }\end{array}$ & $\begin{array}{l}\text { Fe(III) reduction } \\
\text { (Vis) }\end{array}$ & $\begin{array}{l}\mathrm{EP} \\
(\mathrm{UV})\end{array}$ \\
\hline Standard & Mercaptans & $\mathrm{GSH}$ & Cysteine & GSH & MPA & MPA & Sodium sulfide & Sodium sulfide & Sodium sulfide \\
\hline Range $(\mu \mathrm{M})$ & $3-40$ & $1-100$ & $0.2-1.5$ & $1-450$ & $1-200$ & $1-500$ & $1.8-360$ & $1-42$ & $3-250$ \\
\hline $\begin{array}{l}\text { Detection limit } \\
(\mu \mathrm{M})\end{array}$ & 3 & 1 & 0.2 & 1.1 & 0.2 & 0.6 & 1.8 & 1 & 2.8 \\
\hline $\begin{array}{l}\text { Quantification } \\
\text { limit }(\mu \mathrm{M})\end{array}$ & - & - & - & 3.5 & 0.6 & 2.1 & - & - & 7.3 \\
\hline $\begin{array}{l}\text { Reaction time } \\
\text { (min.) }\end{array}$ & 5 & 60 & 60 & 15 & 1 & 2 & 5 & few min. & 15 \\
\hline RSD (\%) & - & - & - & 6.2 & 3.1 & 1.8 & - & 2.5 & 1.9 \\
\hline Reference & [12] & [13] & [15] & [34] & $\begin{array}{c}\text { Method } \\
\text { adapted from } \\
{[7] \text { and [28] }}\end{array}$ & $\begin{array}{l}\text { This } \\
\text { work }\end{array}$ & [16] & [17] & This work \\
\hline
\end{tabular}


2 Table. 2 Recovery of MPA and sulfides in binary solution with potential interfering 3 compounds. $[\mathrm{EP}]=50 \mathrm{mM} ;[\mathrm{MPA}]$ or $\left[\mathrm{HS}^{-}\right]=100 \mu \mathrm{M} ; \mathrm{pH}=9.5$; Reaction time $=2 \mathrm{~min}$. (MPA) 4 or $15 \min \left(\mathrm{HS}^{-}\right)$.

5

\begin{tabular}{lcclcc}
\hline & MPA $\mathbf{( 1 0 0} \boldsymbol{\mu M})$ & \multicolumn{4}{c}{ Sulfides $\mathbf{( 1 0 0} \boldsymbol{\mu M})$} \\
\hline Interfering & & & Interfering & \\
compound & Amount & Recovery & compound & Amount & Recovery \\
\hline Sulfates & $20 \mathrm{mM}$ & $92.40 \%$ & Sulfates & $20 \mathrm{mM}$ & $95.70 \%$ \\
Sulfites & $5 \mathrm{mM}$ & $94.80 \%$ & Sulfites & $5 \mathrm{mM}$ & $93.70 \%$ \\
Methionine & $0.5 \mathrm{mM}$ & $95.20 \%$ & Methionine & $3 \mathrm{mM}$ & $96.70 \%$ \\
Glycine & $1 \mathrm{mM}$ & $93.50 \%$ & Glycine & $1 \mathrm{mM}$ & $91.50 \%$ \\
Ammonium & $20 \mathrm{mM}$ & $98.70 \%$ & Ammonium & $20 \mathrm{mM}$ & $95.60 \%$ \\
Iodide & $50 \mathrm{mM}$ & $98.30 \%$ & Iodide & $50 \mathrm{mM}$ & $98.50 \%$ \\
Chloride & $50 \mathrm{mM}$ & $97.90 \%$ & Chloride & $50 \mathrm{mM}$ & $97.60 \%$ \\
Bromide & $50 \mathrm{mM}$ & $98.90 \%$ & Bromide & $50 \mathrm{mM}$ & $96.70 \%$ \\
Thyocyanate & $50 \mathrm{mM}$ & $97.10 \%$ & Thyocyanate & $50 \mathrm{mM}$ & $96.60 \%$ \\
Acetate & $50 \mathrm{mM}$ & $98.90 \%$ & Acetate & $50 \mathrm{mM}$ & $99.30 \%$ \\
Cu & $160 \mu$ & $98.10 \%$ & Cu & $160 \mu \mathrm{M}$ & $98.20 \%$ \\
$\mathrm{Fe}^{3+*}$ & $180 \mu \mathrm{M}$ & $94.30 \%$ & $\mathrm{Fe}^{3+*}$ & $180 \mu \mathrm{M}$ & $95.10 \%$ \\
$\mathrm{Zn}^{2+*}$ & $160 \mu \mathrm{M}$ & $99.60 \%$ & $\mathrm{Zn}^{2+*}$ & $160 \mu \mathrm{M}$ & $98.40 \%$ \\
\hline
\end{tabular}

